\title{
MARCADORES DEL DISCURSO EN LA NORMA CULTA DE BUENOS AIRES Y DE SEVILLA: ESTUDIO CONTRASTIVO
}

\author{
Juana Santana Marrero \\ Universidad de Sevilla
}

\author{
Claudia Borzi \\ Universidad de Buenos Aires - CONICET
}

RESUMEN: En el presente trabajo se estudian contrastivamente marcadores del discurso en la norma culta de dos ciudades, Buenos Aires (BA) y Sevilla (SE), dentro del Proyecto de la Norma Culta Hispánica “Juan M. Lope Blanch”. Se recolectó un total de 2058 marcadores en BA y 2512 en SE. En términos generales, se percibe la tendencia común a utilizar una forma prototípica para cada uno de los contextos que favorecen las distintas clases de marcadores. Por otro lado, la diferencia dialectal se evidencia en partículas que fueron de uso exclusivo de BA (buenísimo, che, claramente, dale, desde ya, de última) o de SE (ea, pues comentador).

PALABRAS CLAVE: marcadores del discurso, variación, norma culta, Buenos Aires, Sevilla

Discourse markers in the educated norm of Buenos Aires and Seville: a contrastive study

ABSTRACT: This paper undertakes a contrastive study of discourse markers in the educated norm of two cities, Buenos Aires (BA) and Seville (SE), as part of the Project for the Study of the Spanish Educated Norm "Juan M. Lope Blanch". A total of 2058 markers were collected in BA and 2512 in SE. In general terms, we see a common tendency to use a prototypical form in each of the contexts that favor the appearance of different types of markers. Additionally, dialectal variation is attested in those particles used only in $\mathrm{BA}$ (buenísimo, che, claramente, dale, desde ya, de última) or in $\mathrm{SE}$ (ea, pues when it expresses a comment).

KEYWORDS: discourse markers, variation, educated norm, Buenos Aires, Seville

\section{INTRODUCCIÓN}

El gran interés generado en los últimos años por el estudio de los marcadores discursivos ha tenido como resultado una ingente cantidad de investigaciones en las que se ha avanzado en su descripción lingüística. Las líneas de análisis abiertas son múltiples. En esta ocasión destacamos aquellas en las que se ha prestado atención a su rentabilidad en zonas 
geográficas concretas y en grupos sociales específicos. Se trata del estudio de los marcadores del discurso a la luz de las herramientas que nos ofrecen la variación dialectal y la social (Carbonero Cano y Santana Marrero, 2010; Valencia, 2014; Valencia y Vigueras, 2015). En efecto, en esta investigación realizaremos un análisis contrastivo de las partículas empleadas en la norma culta de las ciudades de Buenos Aires (BA) y Sevilla (SE). En concreto, nos centramos en los usos que se dan en los hablantes del sociolecto alto, definidos por un mayor grado de instrucción (preferentemente estudios universitarios), los cuales constituyen un referente importante en ámbitos como las instituciones académicas, la educación o los medios de comunicación.

Si bien abundan los trabajos específicos sobre el empleo de estas partículas en una zona particular (Carbonero Cano y Santana Marrero, 2010), son menos comunes los que adoptan un enfoque contrastivo y se centran en describir las semejanzas y las diferencias entre dos o más comunidades de habla (Santana Marrero, 2015a, 2017). En lo que respecta a la perspectiva diastrática, esta investigación toma como punto de partida la iniciativa llevada a cabo por el Proyecto de la Norma Culta Hispánica “Juan M. Lope Blanch” en la que, siguiendo criterios comunes, se analizaron los marcadores discursivos registrados en 9 ciudades (Valencia, 2014; Valencia y Vigueras, 2015), entre las que se incluyen BA y SE. A partir de ese marco general hemos considerado que sería enriquecedor desarrollar estudios comparativos monográficos que nos permitieran conocer con mayor minuciosidad dónde reside lo común y dónde la diferencia en el uso de estas partículas en dos o más áreas específicas. A este respecto, esta investigación es solo muestra de un planteamiento que puede aplicarse a otras ciudades participantes en los estudios citados.

\section{MARCO TEÓRICO Y METODOLÓGICO}

Para asegurar la validez de los resultados fue preciso establecer unos criterios metodológicos comunes en lo relativo a la caracterización de los marcadores discursivos y a la muestra de habla. Siguiendo las pautas propuestas en Valencia (2014), se partió de los presupuestos teóricos expuestos en Martín Zorraquino y Portolés Lázaro (1999). Estos autores proponen una clasificación en 5 grupos: estructuradores de la información, conectores, reformuladores, operadores argumentativos y marcadores conversacionales. A su vez, todos ellos admiten nuevas subdivisiones. Asumiremos para nuestra exposición esta tipología y, de forma general, la nómina de partículas que consideran dentro de cada uno de los bloques establecidos. Además, incluiremos en el análisis otros marcadores analizados en Borzi (2015) y Santana (2015), ${ }^{1}$ donde el objetivo fijado era localizar los dialectalismos propios de cada zona.

En relación con los marcadores que no están incluidos en Martín Zorraquino y Portolés Lázaro (1999), fueran estos propios solamente de BA o de SE o comunes a ambas zonas, corresponde hacer una aclaración. El objetivo del presente trabajo fue realizar una primera 
comparación partiendo de varias investigaciones previas en las que se siguieron pautas metodológicas similares en cuanto al tipo de partículas analizadas y a su clasificación. A pesar de ello, fue inevitable que se produjeran algunas diferencias que nos llevaron a discutir y acordar criterios diferenciadores que luego aplicamos a la clasificación de los casos registrados. Por ejemplo, se reconsideró el análisis de bueno y descartamos casos como igual y también ordenadores de continuidad, porque solo se buscaron en BA, o sobre todo y por lo menos operadores de refuerzo argumentativo, que solo se buscaron en SE. En efecto, se trataba de unidades que podrían haberse empleado en una u otra área si se hubieran rastreado (lógicamente, habrían tenido que darse las circunstancias comunicativas propicias para que aparecieran en el discurso de los hablantes analizados). En otros casos, sin embargo, la ausencia en una de las ciudades se debió a que eran partículas de carácter local. Este es el aspecto que nos interesa destacar en esta investigación. Es decir, fueron relevantes aquellos marcadores que solo se registraron en una de las dos ciudades consideradas y que, aunque se hubieran buscado en la otra zona explorada, no habrían aparecido ejemplos, en tanto que no forman parte de sus hábitos lingüísticos (fueron casos como buenísimo, dale, desde ya o ea, según veremos más adelante). También señalaremos algunos casos en los que una misma partícula se empleó en ambas ciudades, pero con índices de frecuencia significativamente diferentes.

Las muestras de habla sobre las que basaremos nuestro análisis corresponden a la transcripción de encuestas semi-dirigidas realizadas en dos épocas: BA años 6o (Barrenechea, 1987) y 2012 (material inédito); SE 1972 y 1973 (Lamíquiz y Pineda, 1983) y 2012-2013 (material inédito). Siguiendo una metodología común, se acordó tomar las 5800 primeras palabras de un total de 12 encuestas: 6 de la primera etapa y 6 de la segunda. No obstante, tanto en BA como en SE hubo entrevistas, especialmente de la primera época, que no reunían esos criterios de extensión. En tales casos se completaron tomando fragmentos de otras encuestas del mismo cuerpo de datos realizadas a hablantes con la misma caracterización social. De este modo, para completar los materiales de BA se utilizaron 11 entrevistas recolectadas en los años 60 y 6 recogidas en 2012 (Borzi, 2014, 15), y para la submuestra de SE fueron empleadas 15 encuestas realizadas entre los años 1972 y 1973 y 7 de la etapa de 2012 y 2013 (Santana Marrero, 2014, 279).

Para esta investigación no se diferenciaron de forma sistemática los datos en las dos épocas señaladas, pues dicha variable no se percibió como especialmente relevante en los estudios previos. No obstante, cuando se trate de un dato significativo sí se harán las aclaraciones necesarias relativas a la fecha de la muestra.

En cuanto a la caracterización sociolingüística de los informantes, todos pertenecen al sociolecto alto. A su vez, de acuerdo con las pautas establecidas por el PILEI (Lope Blanch, 1986, 26), se trabaja con tres grupos etarios y con una representación idéntica de hombres y mujeres. 
Para el análisis se combinarán criterios cualitativos y cuantitativos. Los primeros se verán bastante limitados por las propias características de la investigación, donde el objetivo no es describir detalladamente el comportamiento de cada uno de los marcadores localizados, sino reconocer de forma sucinta las funciones para las que se emplearon en cada una de las submuestras. Esto nos permitirá su agrupación en clases y realizar el análisis contrastivo entre ambas ciudades: partículas idénticas o distintas en las dos zonas con el mismo valor discursivo, y funciones compartidas o diferentes en cada una de las áreas. Esta parte cualitativa se refuerza con la faceta cuantitativa, que cobra especial relevancia en nuestro estudio. Los índices de frecuencia globales y parciales nos aportarán una visión real de los marcadores con mayor y menor rendimiento, estableciendo pautas de comportamiento común $\mathrm{y}$ aspectos diferenciadores.

\section{ANÁLISIS GLOBAL DE TODOS LOS MARCADORES}

El cómputo global de marcadores en cada una de las ciudades no arrojó datos significativos (tabla 1). Solo cabría señalar que los hablantes cultos sevillanos aventajaron ligeramente a los bonaerenses en el número de unidades empleadas. ${ }^{2}$

\begin{tabular}{|c|c|c|}
\hline Submuestras & N & $\%$ \\
\hline BA & 2058 & 45 \\
\hline SE & 2512 & 55 \\
\hline TOTAL & \multicolumn{2}{|c|}{4750} \\
\hline
\end{tabular}

Tabla 1. Recuento general

En ambas ciudades se documentaron ejemplos de los 5 grupos de marcadores, con algunos aspectos reseñables en cuanto a su distribución, como puede verse en la tabla 2.

\begin{tabular}{|l|c|c|c|c|}
\hline & \multicolumn{2}{|c|}{ BA } & \multicolumn{2}{c|}{ SE } \\
\hline Clases de marcadores & $\mathrm{N}$ & $\%$ & $\mathrm{~N}$ & $\%$ \\
\hline Estructuradores & 188 & 9 & 726 & 29 \\
\hline Conectores & 468 & 23 & 512 & 20 \\
\hline Reformuladores & 289 & 14 & 372 & 15 \\
\hline Operadores & 235 & 11 & 158 & 6 \\
\hline Conversacionales & 878 & 43 & 744 & 30 \\
\hline \multicolumn{1}{|c|}{ SUBTOTALES } & 2058 & \multicolumn{2}{|c|}{2512} \\
\hline
\end{tabular}

Tabla 2. Resultados según clases de marcadores 
En el plano de las semejanzas, la clase más empleada en las dos áreas fue la de los conversacionales. Este dato no resulta extraño si tenemos en cuenta que su uso guarda una relación estrecha con las características de la muestra analizada: oralidad sin elaboración previa y carácter dialogado. En efecto, las formas que pertenecen a este grupo actúan en el ámbito de la construcción del discurso, simultánea a su recepción, y se sitúan en el entorno de pregunta-respuesta (Martín Zorraquino y Portolés Lázaro, 1999, 4143-4145). El porcentaje obtenido en BA ( $54 \%, 878 / 1622)$, levemente superior al de SE (30 \%, 744/1622), hace pensar en partículas como el metadiscursivo este que, como ya perciben Martín Zorraquino y Portolés Lázaro (1999, 4199), es poco usado en España y prevalece en Hispanoamérica, algo que se confirma ahora estadísticamente para BA.

También resulta llamativa la distinta representación que han tenido los estructuradores de la información en las dos zonas exploradas. Esto puede deberse a la documentación solo en la ciudad andaluza de pues comentador, con una elevada rentabilidad (de hecho, fue el uso más recurrente en esta ciudad, con un porcentaje del $20 \%, 510 / 2512)$, mientras que en BA no se registró ningún caso.

\section{ANÁLISIS PARCELADO SEGÚN CLASES DE MARCADORES}

Para todas las subclases de marcadores se observa una tendencia común: concentración de las subdivisiones funcionales y semántico-pragmáticas establecidas en una o dos partículas que destacan en índices de frecuencia, mientras que el resto ocupa lugares cuantitativamente menos destacados. Así sucedió con primero (ordenador de apertura), después (ordenador de continuidad), además (conector aditivo), entonces (conector consecutivo), o sea (reformulador explicativo), en fin (reformulador recapitulativo), en realidad (operador de refuerzo argumentativo), por ejemplo (operador de concreción), claro (modalidad epistémica), bueno (modalidad deóntica), bueno y mira/mirá/mire (enfocador de la alteridad) y bueno (metadiscursivo). Podríamos afirmar, por tanto, que estos marcadores son preferidos por los hablantes cultos de las ciudades en estudio.

\subsection{Estructuradores de la información}

Los estructuradores de la información organizan los contenidos del discurso. En unos casos se introduce un nuevo comentario (comentadores), en otras ocasiones se distribuyen y se secuencian las partes del texto (ordenadores), y otras veces sirven para añadir segmentos informativos que son secundarios o laterales (digresores) (Martín Zorraquino y Portolés Lázaro, 1999, 4083). Según ya hemos apuntado, este grupo de marcadores presenta índices de frecuencia claramente diferentes entre BA y SE, como se aprecia en la tabla 3. 


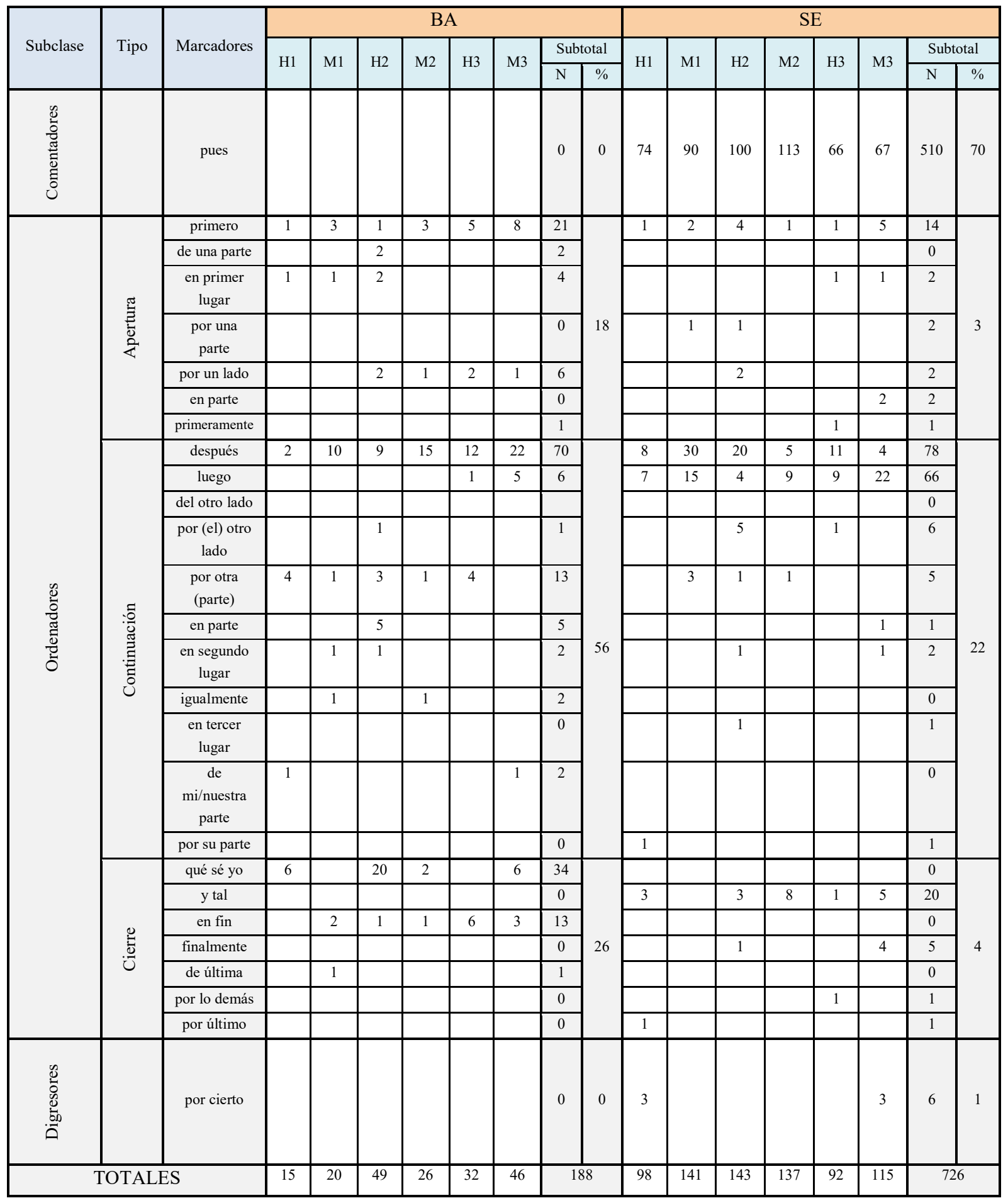

Tabla 3. Estructuradores de la información 
En efecto, junto a una nómina más variada de unidades en SE, con un total de 7 formas distintas que no se emplearon en BA, es reseñable la gran vitalidad que obtuvo pues en el papel de comentador en los hablantes sevillanos, inexistente entre los bonaerenses. Los contextos más recurrentes en los que se registró este marcador fueron: inicio de turno de palabra en el entorno pregunta-respuesta (1); delante del segundo miembro de una construcción bipartita (2) y antes de enunciar el predicado de una secuencia oracional (3); en combinación con eso o nada para indicar cierre con respecto a lo precedente y, al mismo tiempo, introducir un nuevo subtópico (4).

(1) E: ¿Qué edad tenías entonces? // I: Pues en esa... tenía creo que trece años. (SE12M1)

(2) [...] claro, si algún día lo cogían por cualquier cosa, pues todo eso eran pruebas que tenían en contra suya. (SE73M1)

(3) [... y precisamente el joven sevillano es el que sale en una cofradía porque el hombre mayor, pues sí, a lo mejor pertenece a la hermandad pero, naturalmente, no tiene ya o ganas o fuerza física para ponerse durante siete u ocho horas a desfilar delante de un paso. (SE73H1)

(4) [...] es una materia que yo no pensaba nunca que fuera a poder gustarme pero que con mi formación privada, en Derecho Privado, sin embargo, pues al final ha resultado ser una materia bastante bonita y bueno, pues eso, como te digo, soy un abogado entonces bastante generalista. (SE12H1)

Este uso de pues es, por tanto, un rasgo característico de la oralidad no planificada en los hablantes sevillanos del nivel educativo alto. Nuestros datos corroboran la incidencia de este pues en el habla de Sevilla que ya se había constatado en un trabajo previo (Fuentes-Rodríguez, Placencia y Palma-Fahey, 2016) en el que se analizaba el uso de este marcador en tres ciudades hispanohablantes: Quito (Ecuador), Santiago (Chile) y Sevilla (España). En posición inicial, como (1), solo se registraron casos en la ciudad hispalense. Los datos con los que contamos de la norma culta apuntan a que el uso de pues comentador es compartido por otras áreas hispanohablantes. Concretamente, se documentaron registros abundantes en el habla culta de Las Palmas de Gran Canaria, con 390 ocurrencias (Hernández Cabrera, 2015, 264) y en México, con 267 ejemplos (Vigueras Ávila, 2015, 310). Descienden los índices de uso en La Habana, con 33 registros (González Mafud y Perdomo Carmona, 2015, 219), en La Paz, con 7 casos (Mendoza, 2014, 147), en Córdoba-Argentina, también con 7 usos (Toniolo y Zurita, 2015, 131), 3 en Caracas, con 6 ocurrencias (Guirado, 2015, 78), y en Santiago de Chile, con solo 2 registros (Valencia, 2015, 384). 
Continuando con las diferencias, todos los estructuradores localizados en BA se ubicaron en la subclase de los ordenadores, mientras que en SE registramos también ejemplos de comentadores y digresores. Por tanto, las funciones características de esos grupos de marcadores, introducir un nuevo comentario (comentadores) o una información secundaria (digresores), no fueron señaladas explícitamente en BA con las unidades consideradas para esta investigación. 4

Centrándonos ahora en la subclase de los ordenadores, el tipo más destacado fue el de los continuadores. En nuestros materiales, la mayor nómina de partículas de este subtipo pone de manifiesto la importancia que se le da en la oralidad no planificada a indicar la sucesión de los hechos, en detrimento de marcas específicas para el inicio o el cierre, que obtuvieron registros más discretos. En el grupo de los continuadores hay que resaltar la alternancia entre los dos marcadores más frecuentes: luego (5) y después (6).

(5) Porque creo que es un punto importante en la cultura occidental y, en general, en la cultura del mundo conocido, y luego la ciudad es que en sí tiene muchísimo interés, muchísimo interés. (SE12M3)

(6) La excreción de sodio. Después esté... eh... otro tema es el problema de la renina, justamente... (BA6oH2)

Si bien en ambas submuestras después fue la variante más frecuente, característica compartida por otras áreas del mundo hispánico (Santana Marrero, 2015a, 178), destaca el bajo porcentaje de usos de luego en el material de BA (6 \%, 6/106) (Borzi, 2014, 22), sin ejemplos en las entrevistas más recientes. A esto hay que añadir que la mayoría de los casos fueron empleados por hablantes de la tercera generación, un síntoma más de un posible cambio lingüístico en esta comunidad de habla. Sin embargo, en SE luego registró ocurrencias en todos los grupos generacionales y su índice de frecuencia $(41 \%, 66 / 160)$ fue solo ligeramente menor que el de después (49\%, 78/160). Nuestros datos confirman que en la norma culta de la capital argentina la oposición se ha decantado de manera más contundente hacia la variante después, mientras que en la ciudad andaluza ambas formas siguen compitiendo con una gran vitalidad. Esta característica constituye un hecho diferencial en los hábitos lingüísticos de las dos áreas bajo estudio.

También entre los ordenadores, las marcas de cierre fueron poco representativas en ambas submuestras, con escasos ejemplos distribuidos en un pequeño porcentaje de informantes: BA (48), SE (27). Nuestros datos indican que, en términos generales, se trata de una estrategia poco recurrente en la oralidad no planificada, donde existe una mayor tendencia a marcar explícitamente la continuidad. Dentro de este subtipo de marcadores cabe señalar para BA el 
uso de de última (7), que añade el matiz de 'como última opción'. Se trata de un recurso no característico de los sevillanos, quienes no han registrado ningún ejemplo.

(7) Ponele yo soy como muy miedosa, como que manejo hace un montón, pero ni loca me subo a una autopista. Todo el mundo te dice: iay!, pero es re fácil manejar por autopista, o General Paz de última tampoco. (BA12M1)

En la oralidad sin elaboración previa es habitual encontrar fórmulas que aporten al discurso un valor de cierre inespecífico (Loureda Lamas, 2000, 337). Con ellas el hablante indica que no es necesario concretar más o añadir nuevos datos. Para realizar esta función, los informantes bonaerenses se decantaron por qué se yo (8), ausente en el material de SE, mientras que los sevillanos prefirieron la fórmula y tal (9), no utilizada en BA.

(8) I: Bueno, yo creo que lo adquirí desde que nací, porque como desde chica fui al campo y como mi padre se ocupaba de eso tradicionalmente - lo debo haber tenido adentro- y mi abuelo también, y mi bisabuelo también. // E: Claro, es una cosa que ya... // I: Entonces es una cosa que ya-qué sé yo. (BA6oM3)

(9) Ayer, por ejemplo, se cometió... se cumplió una efeméride muy trágica, de un joven que lo mataron, de la forma más absurda, en los jardines de... de Murillo, porque allí se reunía mucha gente joven $y$ tal, a... un poco antes del movimiento de lo que se ha llamado la botellona. (SE12H2)

En el subgrupo de digresores, las diferencias cuantitativas de por cierto entre una y otra ciudad no son atribuibles a la variación dialectal, sino a que los informantes de BA no necesitaron usar esta partícula en sus intervenciones, pues se trata de un recurso propio de las dos zonas exploradas.

\subsection{Conectores}

Siguiendo a Martín Zorraquino y Portolés Lázaro (1999, 4093), diferenciaremos los siguientes grupos de conectores: aditivos (unen argumentos coorientados), consecutivos (marcan la consecuencia con respecto a un segmento previo) y contraargumentativos (enlazan elementos antiorientados). La tabla 4 muestra que la principal semejanza entre las dos ciudades es la coincidencia en el uso del aditivo y del consecutivo más empleados: además y entonces, respectivamente. 


\begin{tabular}{|c|c|c|c|c|c|c|c|c|c|c|c|c|c|c|c|c|c|}
\hline \multirow{3}{*}{ Subclases } & \multirow{3}{*}{ Marcadores } & \multicolumn{8}{|c|}{ BA } & \multicolumn{8}{|c|}{ SE } \\
\hline & & \multirow{2}{*}{$\mathrm{H} 1$} & \multirow{2}{*}{ M1 } & \multirow{2}{*}{$\mathrm{H} 2$} & \multirow{2}{*}{ M2 } & \multirow{2}{*}{ H3 } & \multirow{2}{*}{ M3 } & \multicolumn{2}{|c|}{ Subtotal } & \multirow{2}{*}{$\mathrm{H} 1$} & \multirow{2}{*}{ M1 } & \multirow{2}{*}{$\mathrm{H} 2$} & \multirow{2}{*}{ M2 } & \multirow{2}{*}{ H3 } & \multirow{2}{*}{ M3 } & \multicolumn{2}{|c|}{ Subtotal } \\
\hline & & & & & & & & $\mathrm{N}$ & $\%$ & & & & & & & $\mathrm{~N}$ & $\%$ \\
\hline \multirow{6}{*}{$\stackrel{\substack{0 \\
:}}{:}$} & además & 13 & 26 & 7 & 14 & 7 & 10 & 77 & \multirow{6}{*}{25} & 9 & 20 & 13 & 22 & 7 & 12 & 83 & \multirow{6}{*}{29} \\
\hline & incluso & 4 & 1 & 5 & & 2 & & 12 & & 11 & & 15 & 6 & 9 & 8 & 49 & \\
\hline & inclusive & & 2 & 3 & 5 & 1 & 3 & 14 & & & & & & & & 0 & \\
\hline & aparte & & & 1 & 1 & 4 & & 6 & & 5 & & 1 & 1 & & & 7 & \\
\hline & es más & 1 & & & 3 & 1 & & 5 & & & & 1 & 1 & 1 & 3 & 6 & \\
\hline & encima & & & & 1 & 1 & & 2 & & & 2 & & 2 & & & 4 & \\
\hline \multirow{9}{*}{$\begin{array}{l}\stackrel{0}{0} \\
. \\
0 \\
0 \\
0 \\
0 \\
0\end{array}$} & entonces & 21 & 54 & 42 & 64 & 23 & 37 & 241 & \multirow{9}{*}{60} & 27 & 36 & 45 & 67 & 38 & 26 & 239 & \multirow{9}{*}{55} \\
\hline & así que & 11 & 4 & 5 & 2 & 1 & 6 & 29 & & 4 & 6 & 1 & 2 & & 3 & 16 & \\
\hline & por (lo) tanto & & & & & & & 0 & & 3 & 1 & 2 & & 2 & 12 & 20 & \\
\hline & así (ilustrador) & 1 & & 4 & 1 & 1 & & 7 & & & & & & & & 0 & \\
\hline & así (conclusivo) & & 2 & & 1 & 2 & & 5 & & & & & & & & 0 & \\
\hline & por consiguiente & & & & & & & 0 & & & & & 1 & 2 & & 3 & \\
\hline & pues & & & & & 1 & & 1 & & & & & & 1 & 1 & 2 & \\
\hline & de ahí & & & & & & & 0 & & & & 1 & & & & 1 & \\
\hline & en consecuencia & & & & & & & 0 & & & & 1 & & & & 1 & \\
\hline \multirow{8}{*}{ 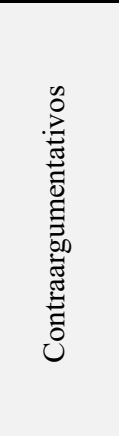 } & sin embargo & 1 & & & 5 & 1 & 1 & 8 & \multirow{8}{*}{15} & 25 & & 11 & 6 & 8 & 3 & 53 & \multirow{8}{*}{16} \\
\hline & ahora & 11 & 2 & 5 & 16 & 11 & 3 & 48 & & & 3 & 2 & 1 & 5 & 1 & 12 & \\
\hline & en cambio & & 2 & & 1 & 2 & 1 & 6 & & & & & 3 & 2 & 3 & 8 & \\
\hline & no obstante & & & & & & & 0 & & 3 & & & 3 & & 1 & 7 & \\
\hline & ahora bien & & & 1 & & 2 & & 3 & & & & & & & & 0 & \\
\hline & por el contrario & & & 2 & 1 & & & 3 & & & & & & & & 0 & \\
\hline & con todo & & & & & & & 0 & & & & & 1 & & & 1 & \\
\hline & eso sí & & 1 & & & & & 1 & & & & & & & & 0 & \\
\hline & OTALES & 63 & 94 & 75 & 115 & 60 & 61 & & & 87 & 68 & 93 & 116 & 75 & 73 & & \\
\hline
\end{tabular}

Tabla 4. Conectores

Además suma segmentos con igual (10) y con diferente (11) fuerza escalar, con lo que se convierte en la forma más frecuente (Martín Zorraquino y Portolés Lázaro, 1999, 4094). Algo parecido sucede con entonces, que Martín Zorraquino y Portolés Lázaro (1999, 4107) entienden como un nexo consecutivo "débil". Es especialmente rentable en contextos narrativos (12), donde los hechos se van enlazando siguiendo criterios de causa-consecuencia y, en ocasiones, siguiendo una sucesión cronológica (se deja así entrever el origen temporal de este marcador). En ambas ciudades entonces fue más rentable. 
(10) Así que sí, toda mi vida en Los Remedios y me considero además de Los Remedios y es un barrio del que estoy orgulloso y encantado de haberme criado aquí porque es un barrio bueno [...] (SE12H1)

(11) Bueno, una película que, por ejemplo, me... me gustó fue “Los monstruos", italiana, muy ágil; porque además me aburren mucho esos diálogos [...] (BA6oH1)

(12) [...] yo cuando empecé con este ritmo me quedaban cuatro materias todavía para recibirme, tenía que ir a la facultad. Entonces yo fui difiriendo muchas cosas que a mí me gustaban. (BA6oH1)

Entre los conectores aditivos señalamos la alternancia inclusive/incluso, que se registró solo en la norma culta de BA (13), la mayoría de los casos en las encuestas antiguas (Borzi, 2014, 26). En SE solo se empleó incluso (14). Esto nos hace pensar que, a pesar de que inclusive tuvo cierta rentabilidad en BA6o, actualmente coinciden ambas urbes en la tendencia a la preferencia de incluso entre los hablantes cultos.

(13) Siempre todas estas investigaciones inclusive la de Ochoa... eh... por supuesto, está basada en miles de otras investigaciones previas [...] (BA60H2)

(14) [...] he tratado muchos temas, unos con más suerte y otras con menos. Incluso también he tenido la suerte de tratar bastantes asuntos de contratación administrativa. (SE12H1)

En la subclase de los conectores consecutivos destaca la escasa presencia en ambas submuestras de pues con este valor (15) y (16). Los ejemplos se localizaron en sujetos de la tercera generación, lo que hace pensar que es una fórmula con cierta tendencia a ir desapareciendo de los hábitos lingüísticos de sus hablantes.

(15) Imagínese que con todos los líos del sesenta y seis y todo eso - por más que a nosotros tanto no nos afectó- pero igual - un poco sí. Y ahora - pues- reanudaríamos. (BA6oH3)

(16) Y por consiguiente, en una totalidad de treinta años o treinta cursos, diferentes asignaturas, como he dicho, de cinco asignaturas, pasando por mis manos, pues, todo el clero sevillano, desde los treinta años. (SE73H3)

En la subclase de contraargumentativos, si nos fijamos en los índices de variación entre sin embargo (17), no obstante (18) y en cambio (19), hay coincidencia en las dos ciudades en que sin embargo fue el más empleado. En la capital andaluza no obstante es el menos utilizado, sin ejemplos en BA. Este último no parece ser un conector característico de este tipo de situación comunicativa, por su escasa documentación. 5 
(17) I: Y eso que, acá tanto la salud como la educación son gratuitas, que en otros lugares de Latinoamérica ninguna de las dos son gratuitas. // E: Claro. // I: Sin embargo sigue habiendo mucha diferencia [...] (BA12M3)

(18) Especialmente en el colegio de las Carmelitas de la Caridad, y, no obstante, también, pues, una especie de profesores y de enseñanzas así, más o menos en casa. (SE73M2)

(19) Claro, y ahí fue donde ella decía que... sí sí, ella iba a esquiar en fin; en cambio nosotros íbamos modestamente a... a buscar un profesor y a ver si nos enseñaba algo. (BA6oM1)

El resto de los conectores utilizados solo en una de las dos ciudades presentaron una escasa rentabilidad. Su presencia o su ausencia parece obedecer a las necesidades comunicativas del hablante y no a la distribución diatópica de la muestra.

\subsection{Reformuladores}

En el ámbito de la reformulación se distinguen marcadores para señalar los procedimientos de la paráfrasis explicativa, la rectificación, el distanciamiento y la recapitulación (Martín Zorraquino y Portolés Lázaro, 1999, 4122).

\begin{tabular}{|c|c|c|c|c|c|c|c|c|c|c|c|c|c|c|c|c|c|}
\hline \multirow{3}{*}{ Subclases } & \multirow{3}{*}{ Marcadores } & \multicolumn{8}{|c|}{$\mathrm{BA}$} & \multicolumn{8}{|c|}{ SE } \\
\hline & & \multirow{2}{*}{ H1 } & \multirow{2}{*}{ M1 } & \multirow{2}{*}{$\mathrm{H} 2$} & \multirow{2}{*}{ M2 } & \multirow{2}{*}{ H3 } & \multirow{2}{*}{ M3 } & \multicolumn{2}{|c|}{ Subtotal } & \multirow{2}{*}{$\mathrm{H} 1$} & \multirow{2}{*}{ M1 } & \multirow{2}{*}{$\mathrm{H} 2$} & \multirow{2}{*}{ M2 } & \multirow{2}{*}{$\mathrm{H} 3$} & \multirow{2}{*}{ M3 } & \multicolumn{2}{|c|}{ Subtotal } \\
\hline & & & & & & & & $\mathrm{N}$ & $\%$ & & & & & & & $\mathrm{~N}$ & $\%$ \\
\hline \multirow{2}{*}{ Explicativos } & o sea & 2 & 6 & 88 & 6 & 5 & 16 & 123 & \multirow{2}{*}{88} & 17 & 31 & 64 & 22 & 25 & 32 & 191 & \multirow{2}{*}{60} \\
\hline & es decir & 63 & 9 & 18 & 9 & 31 & 1 & 131 & & 12 & 2 & 8 & 2 & 2 & 5 & 31 & \\
\hline \multirow{3}{*}{ De rectificación } & más bien & & & 4 & & 2 & 1 & 7 & \multirow{3}{*}{3} & 1 & & 1 & & 1 & 9 & 12 & \multirow{3}{*}{4} \\
\hline & digo & & 1 & & & & & 1 & & 1 & & & & & & 1 & \\
\hline & mejor dicho & & & & & & & 0 & & & & & & & 1 & 1 & \\
\hline \multirow{2}{*}{ De distanciamiento } & de todas maneras & & & 1 & & & 1 & 2 & \multirow{2}{*}{1} & 1 & & & & & & 1 & \multirow{2}{*}{0,2} \\
\hline & de todos modos & & & & 1 & 1 & & 2 & & & & & & & & 0 & \\
\hline \multirow{4}{*}{ Recapitulativos } & en fin & 1 & 12 & & & 3 & 6 & 22 & \multirow{4}{*}{8} & 26 & 2 & 16 & 31 & 5 & 33 & 113 & \multirow{4}{*}{36} \\
\hline & en definitiva & & & & & 1 & & 1 & & & & 4 & & & & 4 & \\
\hline & al fin y al cabo & & & & & & & 0 & & 6 & & & 3 & 1 & 2 & 12 & \\
\hline & total & & & & & & & 0 & & & 1 & & 1 & & 4 & 6 & \\
\hline \multicolumn{2}{|c|}{ TOTALES } & 66 & 28 & 111 & 3 & 43 & 25 & \multicolumn{2}{|c|}{289} & 64 & 36 & 93 & 59 & 34 & 86 & \multicolumn{2}{|c|}{372} \\
\hline
\end{tabular}

Tabla 5. Reformuladores 
Los hablantes cultos de BA y de SE emplearon de forma exclusiva las variantes es decir (20) y o sea (21) para la reformulación explicativa, con ejemplos en todas las casillas sociolingüísticas (tabla 5). No obstante, se aprecian diferencias en cuanto a su rentabilidad. Mientras que en BA hay preferencia por la variante es decir $(80,86 \%, 131 / 162)$, en SE prefieren o sea $(60,83 \%, 191 / 314)$. Junto a esta diferencia, una coincidencia: $o$ sea se ha ido asentando en la norma culta de ambas urbes, especialmente en las encuestas nuevas, donde se aprecia un acusado descenso de es decir: de 120 ocurrencias en BA6o a 11 en BA12 (Borzi, 2014, 30); y de 21 en SE73 a 10 en SE12 (Santana Marrero, 2014, 294). En el caso de BA12, a esto se añade un significativo incremento de $o$ sea (de 4 casos en BA6o a 119 en BA12).

(20) [...] usted va a la cocina y ve las marmitas que le parece que está- por cocinarse en ese momento, es decir, hay sensación de vida, no es una casa fría donde [...] (BA6oM3)

(21) [...] vivías la Feria de... de... de domingo a domingo, o sea, nosotros hacíamos la preferia, la Feria. (SE12M2)

En los recapitulativos, en fin fue el marcador más rentable en las dos submuestras. Sin embargo, la frecuencia absoluta fue claramente superior en SE. Aquí esta partícula muestra una mayor tendencia a la reiteración en el discurso de un mismo informante, con sucesivas recapitulaciones parciales que dan paso a nuevos subtópicos del tema que se desarrolla (22).

(22) Hombre, por supuesto que sí, muchísima diferencia, [...] pero en fin, yo encuentro que los estudiantes en mi época estaban más formados políticamente, menos dirigidos de lo que están ahora, [...] y claro, era una etapa en la que los jóvenes estaban muy implicados en esa ansia de, en fin, alcanzar la democracia que finalmente se consiguió en este país y tal. [...] También es verdad que todos los que tienen una cierta edad piensan de su época de juventud que es la mejor, sin duda alguna, porque en fin, eso está en la condición humana. (SE73M3)

En los recapitulativos, es significativa la ausencia en BA de al fin y al cabo y, principalmente, de total, especialmente si se tiene en cuenta que es un recurso característico de la oralidad (Martín Zorraquino y Portolés Lázaro, 1999, 4137).

\subsection{Operadores argumentativos}

Siguiendo a Martín Zorraquino y Portolés Lázaro (1999, 4140) distinguimos los operadores de refuerzo argumentativo y los de concreción. Este grupo de marcadores tuvo un comportamiento similar en las dos submuestras: predominan las semejanzas tanto en el tipo 
de partículas como en los porcentajes de uso (tabla 6). En ambas áreas fue en realidad el operador más rentable, además, con ejemplos en la mayoría de los informantes.

\begin{tabular}{|c|c|c|c|c|c|c|c|c|c|c|c|c|c|c|c|c|c|}
\hline \multirow{3}{*}{ Subclases } & \multirow{3}{*}{ Marcadores } & \multicolumn{8}{|c|}{$\overline{\mathrm{BA}}$} & \multicolumn{8}{|c|}{ SE } \\
\hline & & \multirow{2}{*}{$\mathrm{H} 1$} & \multirow{2}{*}{ M1 } & \multirow{2}{*}{$\mathrm{H} 2$} & \multirow{2}{*}{ M2 } & \multirow{2}{*}{ H3 } & \multirow{2}{*}{ M3 } & \multicolumn{2}{|c|}{ Subtotal } & \multirow{2}{*}{ H1 } & \multirow{2}{*}{ M1 } & \multirow{2}{*}{$\mathrm{H} 2$} & \multirow{2}{*}{ M2 } & \multirow{2}{*}{ H3 } & \multirow{2}{*}{ M3 } & \multicolumn{2}{|c|}{ Subtotal } \\
\hline & & & & & & & & $\mathrm{N}$ & $\%$ & & & & & & & $\mathrm{~N}$ & $\%$ \\
\hline \multirow{3}{*}{$\begin{array}{l}\text { De refuerzo } \\
\text { argumentativo }\end{array}$} & en realidad & 3 & 22 & 33 & 6 & 6 & 9 & 79 & \multirow{3}{*}{41} & 16 & 11 & 4 & & 5 & 5 & 41 & \multirow{3}{*}{42} \\
\hline & de hecho & 4 & 2 & 7 & & & 3 & 16 & & 10 & 3 & & 5 & 4 & 1 & 23 & \\
\hline & en el fondo & & & 1 & 1 & & & 2 & & & 1 & & & & 1 & 2 & \\
\hline \multirow{3}{*}{$\begin{array}{c}\text { De } \\
\text { concreción }\end{array}$} & por ejemplo & 16 & 15 & 19 & 15 & 23 & 29 & 117 & \multirow{3}{*}{59} & 17 & 9 & 22 & 22 & 12 & 10 & 92 & \multirow{3}{*}{58} \\
\hline & tipo & & 7 & 7 & & & 1 & 15 & & & & & & & & 0 & \\
\hline & en particular & & 5 & 1 & & & & 6 & & & & & & & & 0 & \\
\hline \multicolumn{2}{|c|}{ TOTALES } & 23 & 51 & 68 & 22 & 29 & 42 & \multicolumn{2}{|c|}{235} & 43 & 24 & 26 & 27 & 21 & 17 & \multicolumn{2}{|c|}{158} \\
\hline
\end{tabular}

Tabla 6. Operadores argumentativos

En la función de concreción, habitual en la oralidad sin planificación previa (Fernández Bernárdez, 1994-1995), por ejemplo fue también la forma más común en ambas ciudades. La principal diferencia viene de la mano de la forma tipo (23), solo característica de BA.

(23) Si un alumno me trata de usted, ehh, yo le digo algo, o sea, le digo algo en broma, tipo: che tratame de vos, digamos, ehh como para que afloje con la formalidad digamos. (BA12H2)

Si nos atenemos a los datos presentados en Borzi $(2015,49)$, el empleo de tipo se ha ido asentando en las últimas décadas, pues solo se documentaron casos en las entrevistas más recientes y en informantes de las dos primeras generaciones.

\subsection{Marcadores conversacionales}

Este bloque de marcadores destaca por ser, junto con el de los estructuradores, el que más contribuye a la diferenciación entre las dos submuestras. Por un lado, se registraron partículas distintas en BA y en SE, y, por otro lado, se apreciaron diferentes porcentajes de uso en algunas de las unidades compartidas. Nos detendremos especialmente en el primer fenómeno. En efecto, si bien el total de formas distintas empleadas en las dos ciudades fue prácticamente idéntico (BA: 21/34 y SE: 22/34), se observaron algunos marcadores que solo fueron localizados en una de las dos zonas. Nos centraremos en aquellos casos que puedan arrojar luz sobre la variación diatópica. 


\begin{tabular}{|c|c|c|c|c|c|c|c|c|c|c|c|c|c|c|c|c|c|}
\hline \multirow{3}{*}{ Subclases } & \multirow{3}{*}{ Marcadores } & \multicolumn{8}{|c|}{$\mathrm{BA}$} & \multicolumn{8}{|c|}{ SE } \\
\hline & & \multirow{2}{*}{ H1 } & \multirow{2}{*}{ M1 } & \multirow{2}{*}{$\mathrm{H} 2$} & \multirow{2}{*}{ M2 } & \multirow{2}{*}{$\mathrm{H} 3$} & \multirow{2}{*}{ M3 } & \multicolumn{2}{|c|}{ Subtotal } & \multirow{2}{*}{ H1 } & \multirow{2}{*}{ M1 } & \multirow{2}{*}{$\mathrm{H} 2$} & \multirow{2}{*}{ M2 } & \multirow{2}{*}{$\mathrm{H} 3$} & \multirow{2}{*}{ M3 } & \multicolumn{2}{|c|}{ Subtotal } \\
\hline & & & & & & & & $\mathrm{N}$ & $\%$ & & & & & & & $\mathrm{~N}$ & $\%$ \\
\hline \multirow{12}{*}{$\begin{array}{l}\text { De modalidad } \\
\text { epistémica }\end{array}$} & claro & 34 & 31 & 16 & 29 & 28 & 11 & 149 & \multirow{12}{*}{26} & 12 & 31 & 6 & 62 & 10 & 40 & 161 & \multirow{12}{*}{39} \\
\hline & por supuesto & & 5 & 10 & & 2 & 5 & 22 & & 1 & 5 & 7 & 2 & & 12 & 27 & \\
\hline & naturalmente & & & 4 & & & & 4 & & 25 & 1 & & 3 & 1 & 7 & 37 & \\
\hline & desde luego & & & & & & & 0 & & 5 & 6 & 4 & 15 & & 8 & 38 & \\
\hline & evidentemente & 2 & 2 & 9 & & 4 & & 17 & & & 1 & 3 & & 6 & 1 & 11 & \\
\hline & claramente & 8 & 3 & 9 & & & & 20 & & & & & & & & 0 & \\
\hline & efectivamente & & & 1 & & & & 1 & & 1 & & 2 & & 2 & 3 & 8 & \\
\hline & desde ya & 4 & 1 & & & & 1 & 6 & & & & & & & & 0 & \\
\hline & por lo visto & & & & & & & 0 & & & 2 & & 2 & & 1 & 5 & \\
\hline & $\sin$ (ninguna) duda & & 4 & & & 1 & & 5 & & & & & & & & 0 & \\
\hline & en efecto & & & & & 1 & & 1 & & & & & & & & 0 & \\
\hline & sin duda alguna & & & & & & & 0 & & & & & & & 1 & 1 & \\
\hline & bueno & 5 & 15 & 17 & 19 & 30 & 15 & 101 & & & 1 & & 8 & 2 & & 11 & \\
\hline & buenísimo & & 2 & & & & & 2 & & & & & & & & 0 & \\
\hline & dale & & & 2 & & & & 2 & & & & & & & & 0 & \\
\hline De modalidad & okey & & & 2 & & & & 2 & 12 & & & & & & & 0 & 2 \\
\hline & bien & & & & & 1 & & 1 & & & & & & & & 0 & \\
\hline & ea & & & & & & & 0 & & & 1 & & & & & 1 & \\
\hline & vale & & & & & & & 0 & & & & & 1 & & & 1 & \\
\hline & vamos & & & & & & & 0 & & 25 & 24 & 7 & 34 & 3 & 16 & 109 & \\
\hline & mira/mirá/mire & 5 & 9 & 9 & 9 & 9 & 5 & 46 & & 1 & 1 & 5 & 7 & 7 & & 21 & \\
\hline & hombre & & & & & & & 0 & & 1 & 4 & 13 & 7 & 2 & 3 & 30 & \\
\hline & bueno & 10 & 7 & 14 & 6 & 13 & 9 & 59 & & 2 & 9 & 2 & 6 & 2 & 4 & 25 & \\
\hline Enfocadores de & viste & 1 & 1 & 2 & 5 & 1 & & 10 & 14 & & & & & & & 0 & 26 \\
\hline Ia anteridad & verás & & & & & & & 0 & & & 2 & & & 4 & & 6 & \\
\hline & che & 1 & 1 & 2 & & & & 4 & & & & & & & & 0 & \\
\hline & oye/oiga & & & & & & & 0 & & & 1 & 1 & 2 & & & 4 & \\
\hline & escuchame & & & 2 & & & & 2 & & & & & & & & 0 & \\
\hline & por favor & 1 & & 1 & & & & 2 & & & & & & & & 0 & \\
\hline & bueno & 25 & 30 & 41 & 24 & 48 & 30 & 198 & & 35 & 91 & 11 & 43 & 20 & 22 & 222 & \\
\hline & este & 29 & 29 & 34 & 19 & 70 & 31 & 212 & & 1 & & & & & & 1 & \\
\hline Metadiscursivos & sí & 4 & 2 & & 1 & 2 & 3 & 12 & 48 & 2 & 9 & 5 & 3 & & 4 & 23 & 33 \\
\hline & bien & & & & & & & 0 & & 1 & & & & & & 1 & \\
\hline & ya & & & & & & & 0 & & & & & & 1 & & 1 & \\
\hline TO & TALES & 129 & 142 & 175 & 112 & 210 & 110 & & & 112 & 189 & 66 & 195 & 60 & 122 & & \\
\hline
\end{tabular}

Tabla 7. Marcadores conversacionales 
Como puede comprobarse en la tabla 7, dentro de los marcadores de modalidad epistémica fueron usos exclusivos de BA claramente y desde ya, mientras que desde luego solo se documentó en SE, todos ellos en la misma esfera semántica de la expresión de la evidencialidad (Martín Zorraquino y Portolés Lázaro, 1999, 4149). En relación con claramente (24), en la ciudad bonaerense se percibe un proceso de variación con la forma más extendida de esta subclase en ambas áreas, claro.

(24) [...] Digamos, o sea, y de hecho los felicité y todo. Pero de algunas personas me daría mucha bronca. Sí, claramente. (BA12H2)

Los datos de Borzi $(2015,60)$ muestran que claramente es una variante más reciente en la norma culta de BA, pues los casos registrados solo se dieron en BA12 y en una cantidad nada despreciable para una sola submuestra: 20 ocurrencias. Esto va de la mano de un descenso de claro en las grabaciones nuevas. Cabe añadir que, frente a la presencia de claro en todas las casillas sociolingüísticas de BA, claramente solo fue empleado por las generaciones más jóvenes, por lo que podríamos estar ante un recurso de reciente creación que va avanzando entre los hablantes de menor edad.

Desde ya (25) y desde luego (26) comparten con claro y claramente el contenido semántico-pragmático de 'evidencia', aunque estos se distinguen por presentar el carácter consabido o esperable del enunciado sobre el que influyen con un grado mayor de contundencia. Hay una mayor implicación del hablante con lo que se afirma, se percibe su apreciación personal (Martín Zorraquino y Portolés Lázaro, 1999, 4152; Fuentes Rodríguez, 2009, 104). Se encuentran similitudes formales entre las dos partículas en lo que respecta a su origen: construcción preposicional desde + adverbio temporal, de ahí sus semejanzas semántico-pragmáticas en su funcionamiento como marcadores.

(25) Ehh creo que esos se podrían evitar teniendo más claridad mental. Claro, ocupándose más de uno, estee y desde ya lo de las reglas de tránsito. (BA12M3)

(26) Yo no sé, desde luego, qué solución le van a dar, ni nada, porque el periódico todos los días, eso es de morirte ya de risa [...] (SE73M2)

De las dos formas consideradas, la variante más generalizada es desde luego, documentada en la norma culta de varias ciudades como Caracas (Guirado, 2015, 97), Córdoba-Argentina (Toniolo y Zurita, 2015, 173), La Habana (González Mafud y Perdomo Carmona, 2015, 249), La Paz (Mendoza, 2014, 159), Las Palmas de Gran Canaria (Hernández Cabrera, 2015, 285) y México (Vigueras Ávila, 2015, 346). Por su parte, desde ya es una forma más local que en BA no entró en competencia con desde luego. Cabe señalar a favor de su 
carácter dialectal que no solamente no se localizaron ejemplos en la submuestra de SE, sino que tampoco hay datos sobre su registro en las otras urbes exploradas en Valencia y Vigueras (2015). Además, no aparece recogida en repertorios de marcadores como Martín Zorraquino y Portolés Lázaro (1999), Briz, Pons y Portolés (2008) o Fuentes Rodríguez (2009). Desde el punto de vista cuantitativo, desde luego fue una partícula con una mayor rentabilidad en SE que desde ya en BA.

En los marcadores de modalidad deóntica, encargados de mostrar conformidad o acuerdo con lo expresado por el interlocutor (Martín Zorraquino y Portolés Lázaro, 1999, 4161), hemos documentado 2 usos específicos de BA, buenísimo y dale; y 1 de SE, ea. El escaso índice de apariciones se debe a la naturaleza de nuestros materiales, que no pueden considerarse estrictamente coloquiales.

Bueno fue el recurso más empleado en las dos ciudades para esta función discursiva. Se advierte, sin embargo, una preferencia importante por la elección de esta forma en BA (101 casos), en comparación con SE (11 casos). Con la misma raíz + sufijo aumentativo se empleó en BA buenísimo (27) para reforzar la actitud de acuerdo con el interlocutor. Solo se documentaron 2 ejemplos en una informante de la primera generación en la submuestra más reciente. Habría que estar alerta por si se tratara de un recurso que se ha incorporado y que está avanzando en los últimos años.

(27) I: Sí tal cual como que es una pavada, una pelotudez que hiciste, listo, cagaste, te moriste // E: Bueno, creo que te hice todas las preguntas // I: Bueno buenísimo // E: A ver, sí, bueno eso es todo. (BA12M1)

Es interesante la zona que ocupa en BA el uso de dale (28) como marcador deóntico, donde no se registra vale. Este último se localizó en la norma culta de SE y de Las Palmas de Gran Canaria (Hernández Cabrera, 2015, 285). Los datos apuntan a que dale es una forma de reciente inclusión entre los hábitos lingüísticos de los hablantes cultos bonaerenses, pues solo se documentaron ejemplos en BA12 (Borzi, 2015, 61).

(28) “[...] Vení a tomar unos mates y salís en un rato y yo me voy a dormir”. Y yo había charlado tanto con el pibe que le dije: "Y bueno, está bien, dale". (BA12H2)

Ea (29) solo obtuvo 1 caso en SE. No obstante, podemos afirmar que es una partícula bastante común en esta modalidad de habla, aunque en contextos que requieren mayor espontaneidad que los que recoge nuestra muestra. Funciona para expresar acuerdo, a veces corroborando que lo dicho por el interlocutor coincide con la interpretación del hablante. 
(29) La iba a llamar cuando llegase a Humahuaca... y ya fue como... yo solamente quería escucharte... digo y yo a ti... simplemente quiero saber, ċestáis todos bien?, sí, todos bien, ea, pues ya está... (SE12M1)

Los enfocadores de la alteridad arrojaron datos interesantes para el contraste. Entre los marcadores registrados en ambas ciudades, bueno vuelve a ser el preferido en este contexto, marcando cierto desacuerdo con el interlocutor. Cabe señalar la mayor rentabilidad de este uso en SE, donde se registró casi el doble de casos (109) que en BA (59).

Por otro lado, las formas che y viste fueron exclusivas de BA, mientras que vamos, hombre y verás solo se dieron en SE. Vamos (30), hombre (31) y che (32) no solo muestran acuerdo con el interlocutor sino que, además, presentan la particularidad de actuar en entornos en los que se refuerza su carácter negociador o atenuador y constituyen muestras de cortesía verbal (Martín Zorraquino y Portolés Lázaro, 1999, 4172, 4173, 4176). En términos generales, actúan como un atenuador en su intento de minimizar el efecto sobre el interlocutor cuando se expresa un contenido que no coincide con lo esperable, con las expectativas generadas, o cuando se trata un aspecto que puede resultar polémico. Esta característica se percibe principalmente cuando el contenido de la intervención es controvertido o al hablante le resulta incómodo. En su papel de reforzar la imagen positiva del hablante, che (32) "construye un interlocutor cercano, familiar e, incluso, cómplice” (Borzi, 2016, 21).

(30) pero al ir solo vas un poquito más... más libre. Pero vamos no soy un aventurero suicida ¿eh? (SE12H3)

(31) E: Bien. Y hablando del Departamento de Francés, ¿̇qué te parece tu jefe? // I: Hombre, no sé, verás, qué te digo. (SE73M1)

(32) Depende de quien sea. Si un alumno me trata de usted, ehh, yo le digo algo, o sea, le digo algo en broma, tipo: che tratame de vos, digamos, ehh como para que afloje con la formalidad digamos. (BA12H2)

En el ejemplo (32) se percibe que esta partícula atenúa la distancia de la fuerza imperativa que aporta tratame. En muchos casos, como se puede apreciar arriba, es una partícula reactiva. La bibliografía especializada, como describe Borzi (2016), señala la extensión de esta forma en Argentina y en otros países como por ejemplo Uruguay.

Además del papel de atenuación, vamos (33) se emplea para reforzar la opinión del hablante, poniendo de manifiesto con claridad que lo que se dice se ajusta a su intención comunicativa, y también se utiliza en procesos de explicación, aclaración o precisión (Fuentes Rodríguez, 2009, 346-347). Por otra parte, es común que hombre (34) presente contenido modal, dejando entrever una actitud del hablante de sorpresa, fastidio, resignación, etc. 
(33) E: ¿Te gustan los toros? // I: A mí no. No, a mí me cansan. Yo veo un toro y ya lo he visto para toda la temporada, ¿no? A mí me parece que, que vamos, que es una cosa que como no existe una propia, digamos, voluntariedad por parte del toro a hacer algo distinto y como siempre se sabe a lo que se va, ¿̇no? (SE73H1)

(34) Nueva York era bastante bonita, era como una ciudad medieval con una murallas, muy bien conservadas, que me gustaron mucho, y Edimburgo pues también, me... sorprendió aunque no me gustó porque era muy oscura y hacía mucho frío, pero sí tiene un castillo muy, no sé, muy importante. Hombre, yo sinceramente creo que por el clima, y la gente..., porque esta espontaneidad y esta apertura que tenemos en España es muy agradecida para la gente que viene de paso, y... creo que aquí sí se tratan... bien a la gente de fuera. (SE12M1)

Podemos decir que che (32), vamos (33) y hombre (34) tienen valores semánticos en común, pero no son estrictamente equivalentes, lo que justifica la distinta rentabilidad que han tenido en cada una de las áreas exploradas. Che (32) solo fue localizado entre las generaciones más jóvenes, mientras que los otros dos marcadores son comunes en todas las casillas sociolingüísticas. El tipo de muestra, basada en la encuesta semi-dirigida, puede haber influido también en la escasa presencia de che. En cualquier caso, de nuestros datos se infiere que son partículas que revelan diferencias dialectales entre las ciudades en contraste.

En el ámbito de los enfocadores de la alteridad es habitual utilizar marcadores que se forman a partir de la gramaticalización de verbos de contenido sensorial. Basándose en la raíz léxica ver se han generado los marcadores viste (35) y verás (36), típicos de BA y de SE respectivamente.

(35) [...] tenía por lo menos en esa época una... un Volkswagen, pero viste esos que son... eh... no el... el auto sino la... el furgón y que lo tienen preparado con asientos, ¿no?, como hay algunos acá... eh... para llevar a los chicos al colegio a veces. (BA6oM1)

(36) E: ¿O sea que si le dijera entre... elegir entre vivir en un pueblo y vivir en una ciudad...? // I: Pues... no lo sé... Verás, yo creo que me iría a vivir a un pueblo... no me gusta el aislamiento total... ni mucho menos... (SE12H3)

Partiendo de una base léxica común, hay diferencias en el tiempo del verbo originario: pretérito perfecto simple en BA y futuro en SE. Esto, a nuestro juicio, determina su comportamiento en el discurso: viste alude a lo previo y verás se centra en señalar lo que sigue. En viste (35) se combinan el carácter apelativo definidor de los enfocadores junto con la proyección anafórica, pues se apunta hacia un contenido expuesto previamente como apoyo semántico o como punto de partida para la introducción del nuevo matiz, de una aclaración del mismo tópico que se está desarrollando. Viste busca traer a la conciencia del interlocutor 
algo que se da como conocido y cuya verdad no se discute. Actúa de engarce entre lo previo y lo siguiente. A diferencia de viste, verás (36) tiene proyección catafórica. Se apela al interlocutor para que centre su atención sobre lo que se dice a continuación. Este contenido, a su vez, queda informativamente destacado. Como se aprecia en (36), verás siempre se coloca en posición inicial, precediendo al segmento sobre el que influye, y se percibe una ligera pausa entre el marcador y el segmento que introduce (Fuentes Rodríguez, 2009, 352). El escaso número de ejemplos documentados tanto en el sociolecto alto (Santana, 2015, 470) como en el bajo (Santana Marrero, 2015b, 307) no nos permite hacer una descripción detallada de esta forma. No obstante, en nuestro corpus se constata su utilización en algunos de los contextos señalados por Fuentes Rodríguez (2009, 352). El más común fue el de inicio de respuesta $(67 \%, 4 / 6)$, principalmente en entornos de titubeo (36) o precediendo a una aclaración o explicación (37).

(37) E: Vamos a ver, Emilia, tus padres, ¿dónde nacieron? // I: Pues verás, mi padre nació aquí en Sevilla. Mi madre, no. (SE73M1)

Señalamos un último dato común a los resultados obtenidos de viste y verás: la mayoría de los ejemplos fueron localizados en las encuestas recientes, lo que podría apuntar a que su uso se ha ido asentando y extendiendo en las últimas décadas. Esta tendencia se observó también en las ocurrencias de verás en el sociolecto bajo en la ciudad de Sevilla (Santana Marrero, 2015b, 307).

Finalmente, en el subgrupo de los metadiscursivos, bueno sigue siendo preferido en ambas ciudades. Con este valor se documentaron casos como estrategia para abrir la conversación (38), para recomenzarla después de una interrupción (39) y como estrategia para mantener el tema (40).

(38) I: Bueno, ¿quería preguntarme algo? // E: Bueno, sí... eh... (BA6oM1)

（39） [INTERRUPCIÓN ${ }^{6}$ ] I: bueno, eso sería básicamente / la igualdad / la justicia digamos / la equidad social / estee y el respeto a las leyes. (BA12 $\left.\mathrm{H}_{3}\right)$

(40) [...] hay muchos turistas que vienen de paso y... gente que trabaja para el turismo, como camareros y los dependientes y las tiendas, bueno, mi madre de hecho tiene una tienda en el bajo de nuestra casa y mi familia vivimos arriba [...] (SE12M1)

En último lugar destacamos este, que, aunque empleado en las dos ciudades (41) y (42), presenta diferencias significativas de rentabilidad. 
(41) [...] ehh bueno no todos los días son iguales pero como mi... mi profesión estee la ejerzo en forma liberal... (BA12M3)

(42) [...] mmm... este... Joel Campbell, que estaba en la derecha, se puso en la banda izquierda, donde se había ido Juan Carlos... (SE12H1)

Nuestros datos nos indican que se trata de una partícula característica en la norma culta de BA, 212 casos, y de otras ciudades hispanoamericanas como Córdoba-Argentina, con 338 ocurrencias (Toniolo y Zurita, 2015, 173); Caracas, con 191 ejemplos (Guirado, 2015, 98); o México, con 135 casos (Vigueras Ávila, 2015, 346). Su documentación fue menos relevante en La Habana, con solo 3 ocurrencias (González Mafud y Perdomo Carmona, 2015, 249) y en La Paz, con 4 ejemplos (Mendoza, 2014, 159). A diferencia de BA, este no es una estrategia habitual entre los sevillanos cultos, con solo 1 registro. Como se dijo, nuestros datos confirman las palabras de Martín Zorraquino y Portolés Lázaro (1999, 4199), quienes consideran esta forma más común en Hispanoamérica. Su principal función es rellenar el espacio de tiempo que se produce mientras el hablante busca la expresión que se acomoda a su intencionalidad comunicativa o redireccionar temáticamente la conversación. El silencio implicaría perder el turno de palabra. Eh es una fórmula muy habitual para realizar esta función en SE, según pudimos comprobar en los datos de las encuestas recientes. ${ }^{7}$ En BA esta forma entra en competencia con este, con un índice de usos significativamente superior (Borzi, 2015, 61).

\section{CONCLUSIONES}

El estudio contrastivo de los marcadores en el habla culta de BA y de SE ha revelado semejanzas y diferencias entre ambas ciudades. Entre los ámbitos comunes cabría señalar la coincidencia en la mayoría de los marcadores más empleados para cada clase. En efecto, algunos de ellos constituyen una especie de partículas prototípicas dentro de su entorno semántico-pragmático y funcional, que registraron índices de frecuencia destacados en las dos áreas urbanas bajo estudio, y es posible que también comunes a otras zonas del mundo hispánico. Tal fue el caso de primero (ordenador de apertura), después (ordenador de continuidad), además (conector aditivo), entonces (conector consecutivo), o sea (reformulador explicativo), en fin (reformulador recapitulativo), en realidad (operador de refuerzo argumentativo), por ejemplo (operador de concreción), claro (modalidad epistémica), bueno (modalidad deóntica), bueno y mira/mirá/mire (enfocador de la alteridad) y bueno (metadiscursivo).

Pero también documentamos unidades solo específicas de una de las dos ciudades. A veces esos marcadores son compartidos por otras áreas del mundo hispánico (pues, vamos, hombre, verás), pero no faltan los casos en los que nuestros datos apuntan a que son formas de carácter 
dialectal, bien bonaerenses (de última, desde ya, che, claramente, dale, buenísimo), o bien sevillanas (ea). Fue habitual que estos recursos más locales (tipo, claramente, buenísimo, dale) se emplearan exclusiva o principalmente en las encuestas recientes y en ocasiones en las generaciones más jóvenes, aspecto que nos hablaría de un cambio en curso. Confiamos en que ulteriores estudios contrastivos nos permitan tener nuevos datos para seguir profundizando en estos fenómenos.

\section{NOTAS}

1 Para esta ciudad tuvimos en cuenta los listados de Briz et al. (2008) y de Fuentes Rodríguez (2009).

2 Una diferencia en el mismo sentido se registró si comparamos los totales de Borzi (2015) con los de Santana (2015).

3 A diferencia de lo que ocurre en Buenos Aires, resultan interesantes los casos registrados en Córdoba (Argentina), una ciudad que, como señalan las autoras, los investigadores han considerado siempre como un área geográficamente central, de transición entre las distintas subáreas dialectales del país.

4 Borzi $(2015,33)$ registra en contexto de digresión un uso de perdón, forma rastreada, pero no registrada en Sevilla ni en ese contexto ni como enfocador de la alteridad.

5 Pensamos, incluso, que no obstante podría ser una marca condicionada por el grado de instrucción de los hablantes, ya que tampoco se documentaron ejemplos en una muestra del sociolecto bajo (Santana Marrero, 2015b).

6 Durante el desarrollo de la entrevista, hubo un breve corte de la grabación. A continuación se reanudó el diálogo.

$7 \quad$ No incluimos este marcador en el recuento porque no se transcribió en las encuestas antiguas. Para homogeneizar criterios, se excluyó también del cómputo de BA.

\section{REFERENCIAS BIBLIOGRÁFICAS}

BARRENECHEA, A. M. (coord.). 1987. El habla culta de la ciudad de Buenos Aires. Materiales para su estudio. Tomos I y II. Buenos Aires: Facultad de Filosofía y Letras, Universidad de Buenos Aires.

BORZI, C. 2014. "Marcadores del discurso de Buenos Aires", en Valencia, A. (coord.), Marcadores discursivos en la norma culta hispánica: 1964-2014. Cuadernos de la ALFAL, 5, pp. 13-42.

BORZI, C. 2015. "Marcadores discursivos de Buenos Aires", en Valencia, A. y Vigueras, A. (coords.), Más sobre marcadores hispánicos. Usos de España y América en el corpus de estudio de la norma culta. México, D. F.: Universidad Nacional Autónoma de México, pp. 19-67.

BORZI, C. 2016. "El che argentino: sus contextos de uso y su significado", Philologia Hispalensis, 30(1), pp. 9-32. 
BRIZ, A., PONS, S. y PORTOLÉS, J. 2008. Diccionario de partículas discursivas del español. Disponible en www.dpde.es.

CARBONERO CANO, P. y SANTANA MARRERO, J. 2010. "Los marcadores y la variación espacial y social”, en Loureda Lamas, Ó. y Acín Villa, E. (coords.), Los estudios sobre marcadores del discurso del español, hoy. Madrid: Arco/Libros, pp. 497-521.

FERNÁNDEZ BERNÁRDEZ, C. 1994-1995. "Marcadores textuales de ‘ejemplificación' textual”, Estudios de Lingüística. Universidad de Alicante, 10, pp. 103-144.

FUENTES RODRÍGUEZ, C. 2009. Diccionario de conectores y operadores del español. Madrid: Arco/Libros.

FUENTES-RODRÍGUEZ, C., PLACENCIA, M. E. y PALMA-FAHEY, M. 2016. "Regional pragmatic variation in the use of the discourse marker pues in informal talk among university students in Quito (Ecuador), Santiago (Chile) and Seville (Spain)”, Journal of Pragmatics, 97, pp. 74-92.

GONZÁLEZ MAFUD, A. M. y PERDOMO CARMONA, M. 2015. "Marcadores discursivos de La Habana”, en Valencia, A. y Vigueras, A. (coords.), Más sobre marcadores hispánicos. Usos de España y América en el corpus de estudio de la norma culta. México, D. F.: Universidad Nacional Autónoma de México, pp. 207-255.

GUIRADO, K. 2015. "Marcadores discursivos de Caracas”, en Valencia, A. y Vigueras, A. (coords.), Más sobre marcadores hispánicos. Usos de España y América en el corpus de estudio de la norma culta. México, D. F.: Universidad Nacional Autónoma de México, pp. 69-122.

HERNÁNDEZ CABRERA, C. E. 2015. "Marcadores discursivos de Las Palmas de Gran Canaria”, en Valencia, A. y Vigueras, A. (coords.), Más sobre marcadores hispánicos. Usos de España y América en el corpus de estudio de la norma culta. México, D. F.: Universidad Nacional Autónoma de México, pp. 257-301.

LAMÍQUIZ, V. y PINEDA, M. Á. (eds.). 1983. Encuestas del habla urbana de Sevilla. Nivel culto. Sociolingüística Andaluza 2. Sevilla: Publicaciones de la Universidad de Sevilla.

LOPE BLANCH, J. M. 1986. El estudio del español hablado culto. Historia de un proyecto. México, D. F.: Universidad Nacional Autónoma de México.

LOUREDA LAMAS, Ó. 200o. "Sobre un tipo de marcadores discursivos de enumeración en el español actual”, RILCE. Revista de Filología Hispánica, 16(2), pp. 325-342.

MARTÍN ZORRAQUINO, M. A. y PORTOLÉS LÁZARO, J. 1999. “Los marcadores del discurso”, en Bosque, I. y Demonte, V. (dirs.), Gramática descriptiva de la lengua española. Vol. 3. Madrid: Espasa Calpe, pp. 4051-4213.

MENDOZA, J. G. 2014. “Marcadores discursivos en La Paz”, en Valencia, A. (coord.), Marcadores discursivos en la norma culta hispánica: 1964-2014. Cuadernos de la ALFAL, 5, pp. 140-162.

SANTANA, J. 2015. "Marcadores discursivos de Sevilla”, en Valencia, A. y Vigueras, A. (coords.), Más sobre marcadores hispánicos. Usos de España y América en el corpus de estudio de la norma culta. México, D. F.: Universidad Nacional Autónoma de México, pp. 443-490.

SANTANA MARRERO, J. 2014. "Marcadores del discurso de Sevilla", en Valencia, A. (coord.), 
Marcadores discursivos en la norma culta hispánica: 1964-2014. Cuadernos de la ALFAL, 5, pp. 277-311.

SANTANA MARRERO, J. 2015a. "Luego and después with a textual function: discursive use and variation in the Panhispanic educated norm”, Revista de Lenguas para Fines Específicos, 21(1), pp. 160-183.

SANTANA MARRERO, J. 2015b. "Marcadores del discurso y variación diastrática: estudio en el sociolecto bajo”, en Santana Marrero, J. (dir.), León-Castro Gómez, M. y Zerva, A. (eds.), La variación en el español actual. Estudios dedicados al profesor Pedro Carbonero. Sevilla: Editorial Universidad de Sevilla, pp. 289-313.

SANTANA MARRERO, J. 2017. "Marcadores interrogativos de interacción conversacional en la norma culta hispánica”, en 90 años de la Academia Boliviana de la Lengua. La Paz: Academia Boliviana de la Lengua Española, pp. 232-286.

TONIOLO, M. T. y ZURITA, M. E. 2015. "Marcadores discursivos de Córdoba, Argentina", en Valencia, A. y Vigueras, A. (coords.), Más sobre marcadores hispánicos. Usos de España y América en el corpus de estudio de la norma culta. México, D. F.: Universidad Nacional Autónoma de México, pp. 123-206.

VALENCIA, A. (coord.). 2014. Marcadores discursivos en la norma culta hispánica: 1964-2014. Cuadernos de la ALFAL, 5 .

VALENCIA, A. 2015. "Marcadores discursivos de Santiago de Chile", en Valencia, A. y Vigueras, A. (coords.), Más sobre marcadores hispánicos. Usos de España y América en el corpus de estudio de la norma culta. México, D. F.: Universidad Nacional Autónoma de México, pp. 377-441.

VALENCIA, A. y VIGUERAS, A. (coords.). 2015. Más sobre marcadores hispánicos. Usos de España y América en el corpus de estudio de la norma culta. México, D. F.: Universidad Nacional Autónoma de México.

VIGUERAS ÁVILA, A. 2015. "Marcadores discursivos de la ciudad de México", en Valencia, A. y Vigueras, A. (coords.), Más sobre marcadores hispánicos. Usos de España y América en el corpus de estudio de la norma culta. México, D. F.: Universidad Nacional Autónoma de México, pp. 303-376.

\section{NOTA SOBRE LAS AUTORAS}

Juana Santana Marrero es profesora Titular del área de Lengua Española en la Universidad de Sevilla, España. ORCID: 0000-0002-7715-0141

Claudia Borzi es Investigadora Principal del Consejo Nacional de Investigaciones Científicas y Técnicas y profesora Asociada Regular con cátedra a cargo en la Universidad de Buenos Aires, Argentina. 